\title{
A low-cost system for measuring and analyzing human movement in three dimensions
}

\author{
PATRICK HAGGARD \\ University College London, London, England
}

\begin{abstract}
This paper describes a system for measuring the three-dimensional movement of a point on the human body. The core components of the system are an ultrasonic computer mouse (The OWL), an IBM-compatible personal computer, and a suite of recording and analysis software written in National Instruments' LabVIEW instrumentation programming language. The cost of these components is sufficiently low that we have been able to create a system for measuring human kinematics that is within the reach of a typical budget for student practical classes.
\end{abstract}

Knowledge of the psychological representations and information processing underlying the control of human movement has historically been limited by the difficulty of quantitative measurement of human kinematics. Recording of unconstrained human arm and body movement only became feasible, some decades ago, with the introduction of optical tracking technology using active infrared transmitting markers. These systems involve CCD cameras located in precalibrated or user-calibrated positions, which detect the successive strobing of a number of infrared emitting diodes, and then use calibration data to transform the location of the diodes from camera focal plane coordinates to absolute locations in space. The cost of optical systems of this kind, such as Selspot and Optotrak, is high, and they therefore remain specialist tools used for advanced research programs in dedicated centers. In particular, most teaching institutions will be unable to afford the technological investment required to demonstrate movement recording to students. Most psychology students, therefore, have no opportunity to measure, record, and analyze human kinematic data. Clearly, a simple low-cost method of measuring human movement would enhance the interest and value of motor skills teaching in a whole range of undergraduate disciplines.

The most obvious opportunity for recording movement for these purposes is the ubiquitous computer mouse. It is cheap, widely available, easy to use, and has excellent software support. However, the standard computer mouse has several limitations as a method of quantitative movement recording. First, motions must be in a plane and must be in contact with a firm surface in order for the mouse's trackball to rotate. Second, imperfect traction between the surface, the trackball, and the shaft encoder wheels means that physical movements of the mouse are neither necessary nor sufficient for a motion signal to be detected by the host computer. Third, the axes of computer mice are de-

Correspondence should be addressed to P. Haggard, Department of Psychology, University College London, Gower Street, London WC1E 6BT, England (e-mail: p.haggard@ucl.ac.uk). fined by the orientations of the shaft encoders within the mouse itself; therefore, they do not correspond to the axes of absolute space. As a result, the calculation of direction of mouse movement at any time depends on the current mouse orientation, which cannot be sensed.

The increasing popularity of computer games has generated new low-cost methods for communicating hand movement signals to a host computer. Some of these, such as joysticks, have the benefit of quantitative precision and direct correlation to positions in absolute space, yet still permit only constrained motions. This paper reports an alternative approach using a wireless ultrasonic threedimensional (3-D) mouse to record human movement signals. While sonic and ultrasonic movement tracking has been proposed before, the system described here is characterized by its low cost and ease of use. National Instruments' LabVIEW is used to record movements and to provide visualization and analysis of data. LabVIEW is used because its intuitive and simple programming allows even less technically advanced users to customize software according to their needs. Therefore, the resulting package is flexible: Students with only minimal programming experience can adjust the details of data collection to acquire appropriate kinematics, write their own routines for kinematic signal processing, and incorporate their own routines for calculating informative outcome measures (e.g., movement duration, peak velocity, power spectra) to characterize motor performance. As a result, the repertoire of possible analyses is more flexible than that provided by some more expensive packages aimed at the research market. Furthermore, added educational value arises from the students' involvement in the technical aspects of human movement analysis, such as sampling and smoothing frequencies, calibration, and so on.

\section{Hardware Considerations}

The OWL is a wireless, mouse-compatible 3-D pointing device aimed primarily at the computer gaming industry. It consists of a lightweight ring (worn on the index finger) and a base unit. The ring contains an ultrasonic transmitter whose signals are detected by three ultrasonic 


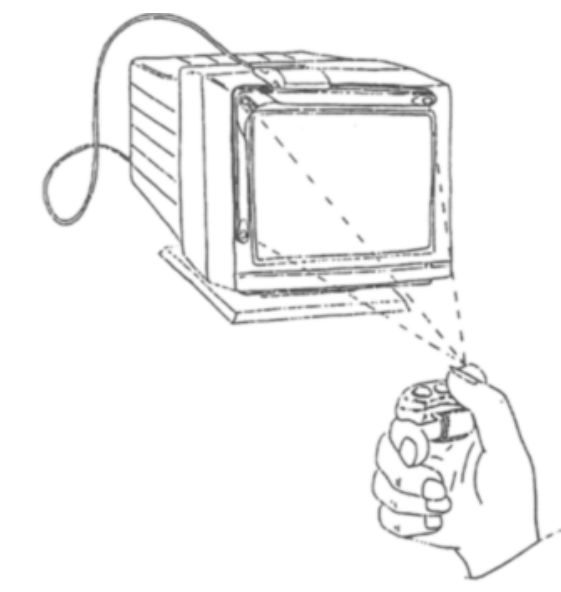

The OWL base unit and ring shown in proper operating position.

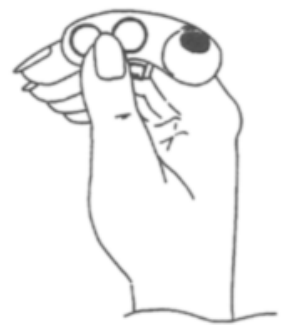

Proper position of ring on hand.

Figure 1. The components of the OWL device. Note the three ultrasonic receivers mounted in an $L$-shaped frame, here positioned on a computer monitor (upper panel), and the ultrasonic transmitter and two mouse buttons, here worn on the index finger (lower panel). From The OWL User's Manual (4th ed., pp. 8-9), 1996, Tel Aviv: Pegasus Technologies Ltd. Copyright 1996 by Pegasus Technologies Ltd. Reprinted with permission.

receivers mounted in the base unit. Differences in the signals received by the three receivers allow the 3-D position of the transmitter to be calculated with respect to the axes defined by the location of the receivers in the base unit. The base unit transmits mouse position coordinates to the computer via the serial port at a rate of $50-58 \mathrm{~Hz}$ (manufacturer's figures), according to conditions. In practice, we have found that these slight variations in sample rate can easily be eliminated by measuring the time at which each sample is collected by the computer's system clock and interpolating the data if necessary to obtain an equally spaced time series (see Software Support section, later). The claimed resolution of this information is below $1 \mathrm{~mm}$, and the working volume is approximately $1 \mathrm{~m}^{3}$. The working volume begins approximately $30 \mathrm{~cm}$ in front of the receiver unit. Two micro switches are also mounted on the ring, adjacent to the ultrasonic transmitter. These transmit logic signals to the base unit via infrared to duplicate the normal functions of the $\mathrm{PC}$ mouse button. The components of the system are shown in Figure 1.

The cost of the OWL device at the time of writing is $\$ 99$. While aimed primarily at the recreational computing market, the OWL is clearly well suited for low-cost measurement of the kinematics of a single point of the human body (e.g., experiments to measure arm movement and postural sway). Indeed, its technical specifications are comparable to other kinematic recording systems aimed at the research market, such as the Flock of Birds and Fastrak.

We have performed a variety of tests to validate the OWL hardware for human movement recording. First, we have assessed the accuracy of the system using a method adapted from Haggard and Wing (1990). These authors suggested that the mean and standard deviation of the reconstructed distance between two markers were crucial statistics for quantifying the accuracy of kinematic recording systems. The mean reconstructed distance is meaningful only in calibrated systems that make absolute position measurements by comparing marker positions with the stored dimensions of a reference frame. This concept does not apply to systems such as the OWL, which report uncalibrated data. However, the variability measures proposed by Haggard and Wing remain relevant. In an accurate system, the reconstructed distance between the two positions should show little variation across workspace locations and across repetitions. Since only one transmitter may be used with the OWL device, we have adapted Haggard and Wing's original method of two markers on a rigid body, by moving the OWL between fixed locations and calculating variations in the reported distance between those locations. We found that the paperfeed function on a standard desktop printer provides a convenient way of translating the transmitter through a fixed distance. We now report three tests of different aspects of the system's accuracy.

The first test measured variations in the reported distance of a movement repeated once at each of four locations in the workspace (Figure 2). These locations correspond to the typical central working area when the OWL receiving unit is mounted on the upper left of a VDU screen. This is the position for which it is designed (Figure 1). Calibration was performed by scaling the mean of all four reported movements to the distance measured with a ruler $(48 \mathrm{~mm})$. The standard deviation of these four numbers gives an estimate of the device's spatial distortion within this working area: The value was $0.44 \mathrm{~mm}$.

In a second test, we repeated the $48-\mathrm{mm}$ movement 10 times at a single workspace location approximately in the center of the four locations shown in Figure 1. These 10 measurements had a coefficient of variation of $0.61 \%$. We used the same calibration method described for the first test above to transform the raw measures of amplitude into 10 calibrated distances. This showed that the standard deviation of these 10 distances was $0.30 \mathrm{~mm}$. This figure gives an estimate of measurement noise in the system. 


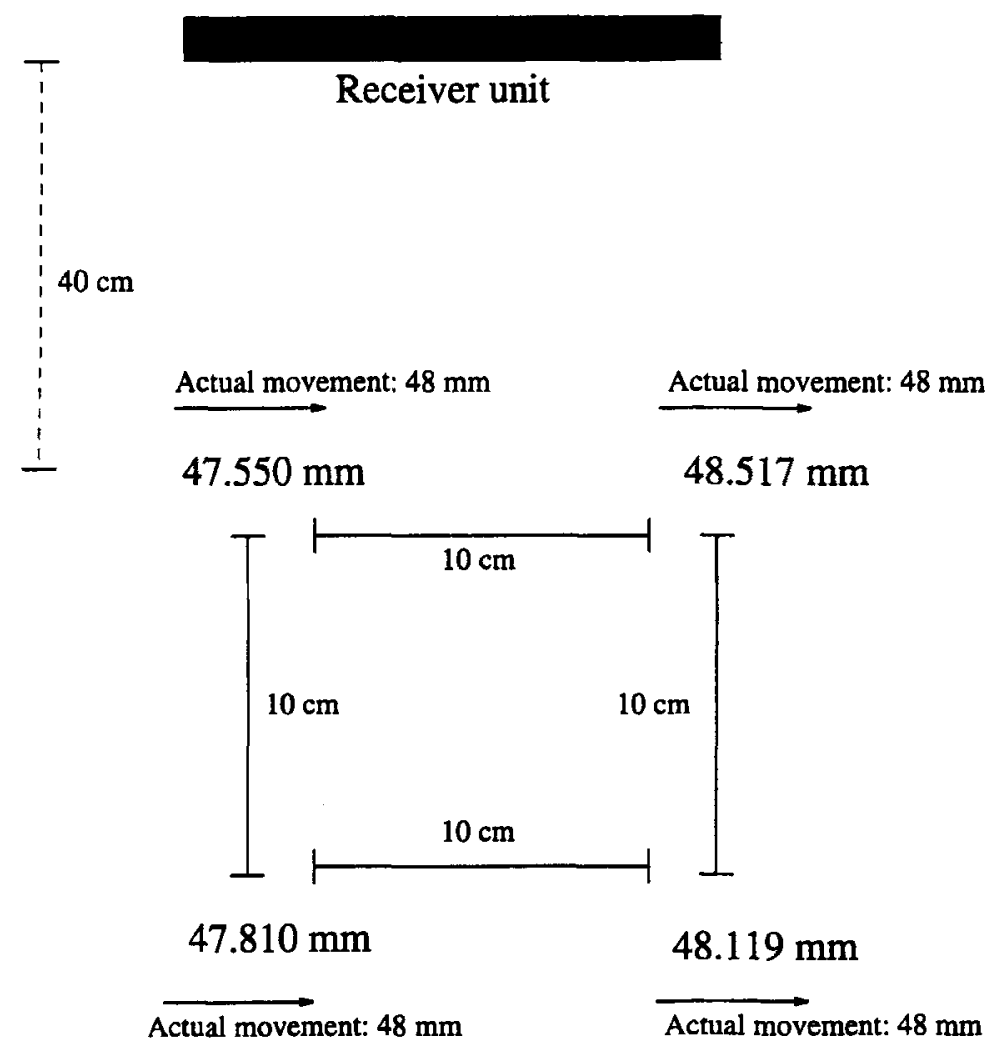

\begin{abstract}
Figure 2. Accuracy of reconstructing a known distance $(48 \mathrm{~mm})$ at four locations within a typical experimental workspace. The displacements were achieved using the paper-feed function on a desktop printer and were always from left to right. The distances between the various movement locations and the base unit are shown. The number in each location gives the reconstructed distance. The OWL was calibrated using the average of these distances; therefore, the variability among the four distances is of more interest than their actual values.
\end{abstract}

In a third test, we measured two 48 -mm displacements at identical workspace locations, but with different objects in the immediate environment of the transmitter, to assess the robustness of motion signals in the face of environmental changes likely to occur in typical movement recording situations. We found that placing a cardboard box, a glass, and an extended hand simultaneously within $10 \mathrm{~cm}$ of the transmitter, but without occluding it, increased the reported distance of the $48-\mathrm{mm}$ motion by $2.92 \%$ ( $1.4 \mathrm{~mm})$.

Finally, we have found it convenient to use the procedure described in these tests (i.e., moving the OWL between two locations a known distance apart) in order to calibrate the OWL. The reported distance in raw data units is simply divided by the physical distance measured with a ruler to give a calibration coefficient, which can then be applied in postprocessing software (see later).

To conclude this section, positional measurements made with the OWL device appear to be positionally accurate to less than $1 \mathrm{~mm}$, making them compare favorably with other tracking systems. However, the immediate physical environment of the receiver should be maintained as constant as possible throughout an experiment in order to avoid the larger distortions of measurement that these may cause. At the same time, it is worth emphasizing what the OWL device cannot do. It cannot track the position of more than one point on the body. It cannot sample at the high frequencies required to study the derivatives of very fast movements-particularly, acceleration profiles. It cannot provide measurements when the transmitter is occluded from the receiver unit.

\section{Software Considerations}

Human movement kinematics generate richer, more complex data than the simple univariate measures, such as error rates or reaction times, that undergraduate psychology students may be used to handling in other areas of psychology. Therefore, it is important to expose students to some of the signal processing and analysis issues that arise when dealing with kinematic data. This makes for a richer learning experience than "point and click" recipes for preprepared experiments. The choice of appropriate software for handling kinematic signals becomes vital when deep learning of kinematic methods is desired. The software system must be sufficiently simple for students to attempt their own quantitative analysis, sufficiently 
complex to allow them to make useful measurements, and sufficiently high level for them to manipulate data without a detailed technical knowledge of the software or a prolonged learning period. We have therefore selected LabVIEW as the software package for data acquisition and analysis for these practical classes. LabVIEW is a graphical programming language specifically aimed at the instrumentation market. Users program computations by creating a front panel where values can be input into controls and results can be read from indicators, and they process data by connecting inputs and outputs to icons representing functions (called virtual instruments in LabVIEW ${ }^{1}$ ) using a graphical wiring tool. In essence, LabVIEW is programmed by drawing flow charts. We have found LabVIEW convenient for undergraduate experimentation. First, the learning curve is rapid: An undergraduate student can typically program a simple LabVIEW operation after $30 \mathrm{~min}$ of training. Second, LabVIEW is unusually powerful given its ease of use: Most operations that can be performed in $\mathrm{C}$ can be performed in LabVIEW. Third, a lowcost student edition of LabVIEW is available to allow students to develop their own work.

\section{Interface Considerations}

Communication between LabVIEW and the OWL is achieved by calling a library (DLL) supplied by the man- ufacturers of the OWL, which reads the current position of the transmitter via the serial port. The aim of kinematic recording is to generate a time series of positions of an effector at equally spaced intervals during the movement. The sampling rate supported by the OWL varies between 50 and $58 \mathrm{~Hz}$ (manufacturer's figures), according to the conditions of the movement. This sampling rate may go down, causing one or more samples to be lost, for two distinct reasons. First, an extremely fast movement of the OWL may temporarily reduce the sampling rate. Other movement recording technologies, such as electromagnetic graphics tablets exhibit similar behavior. Second, the software execution speed may change during the course of a trial. For example, one or more samples may be lost if the operating system overhead suddenly increases, as when the user inadvertently moves or resizes a window or even uses the mouse during the course of data acquisition.

In practice, these limitations do not affect typical data acquistion projects significantly. We have found that a sampling rate of $56 \mathrm{~Hz}$ can be sustained throughout a typical aimed arm movement on a computer with typical specification (486DX66, $8 \mathrm{MB}$ of RAM), and neither the OWL sampling time nor the software execution time are likely to interfere with the sampling rate. The temporal resolution of the system is then comparable to that of more expensive video-based systems. However, the tem-

\section{RUNOWL.VI}

\section{Press < cntrl> R, then Start and Stop buttons}

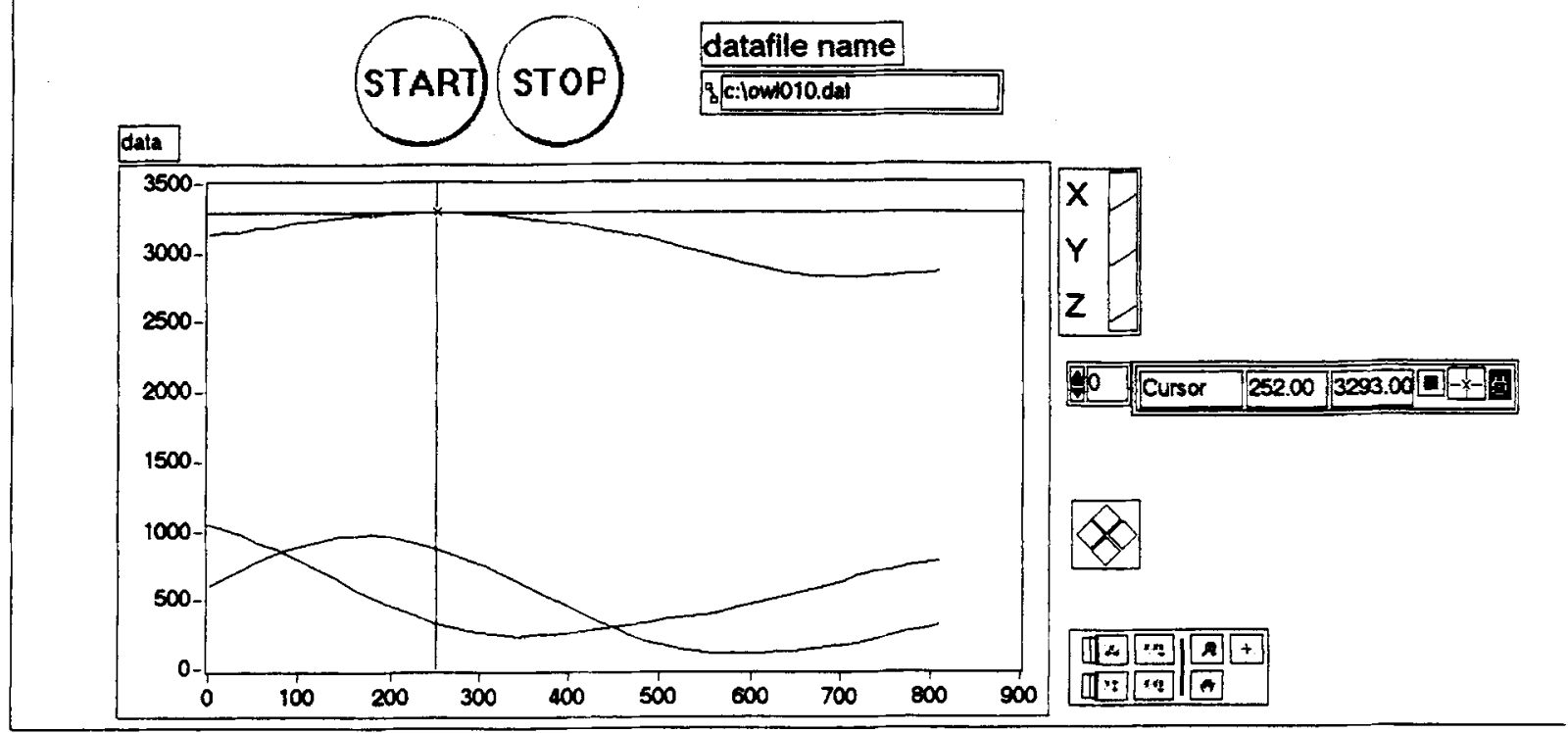

Figure 3. Front panel of a LabVIEW program for acquiring kinematic data using the OWL. Note the simple instructions on the screen, to help unskilled users. 
poral performance of this system, unlike more expensive real-time systems, is not guaranteed, and it depends on the time at which the DLL is called to acquire each sample, rather than on specialized clocking hardware. For this reason, we always record the time by the computer system clock at which each sample is read using the DLL, and we check whether these samples are all separated by the same time interval. We have written a number of programs (see Software Support section below) to interpolate any lost datapoints so that trials with with unevenly sampled data may still be analyzed.

\section{Software Support}

We have written a number of virtual instruments in LabVIEW for performing the basic operations needed to acquire, record, analyze, and interpret human armmovement data in the context of an undergraduate practical laboratory class. These include utilities to verify the hardware (checkOWL.vi) and to display acquired traces (viewOWL.vi). More importantly, we have designed data acquisition and data analysis instruments in LabVIEW so that they can be used to acquire and analyze kinematic data with minimal training, yet support quite complex acquisition and analysis. These are now described in turn, and a discussion of their design principles and value as teaching instruments is also given.

Data collection: runOWL.vi. This program collects 3-D position information from the OWL for a period determined by the experimenter, displays $x, y, z$ waveforms, and stores them on disk as a human readable spreadsheet file, with a filename specified by the experimenter. The sampling rate is set at the OWL's typical rate of $56 \mathrm{~Hz}$. The data acquisition is controlled by start and stop buttons on the front panel of the instrument. In order to minimize possible operating system overhead due to excessive mouse movement during data acquisition, it is often preferable to control these buttons via function keys to which they have been linked: The user presses F1 to start data acquisition, and F2 to stop it. Instructions for basic operation of the program are included on the front panel so that an inexperienced user can collect data without reading lengthy documentation. Figure 3 shows the front panel of the program after collecting one trial.

Data analysis: OWLstat.vi. This program implements some basic signal processing operations appropriate for most kinematic data and allows interactive measurement of the kinematic data. It is also designed to be customizable. A user with minimal LabVIEW program-

\section{OWLSTAT.VI}

\section{1/17/96 09:05 PM}

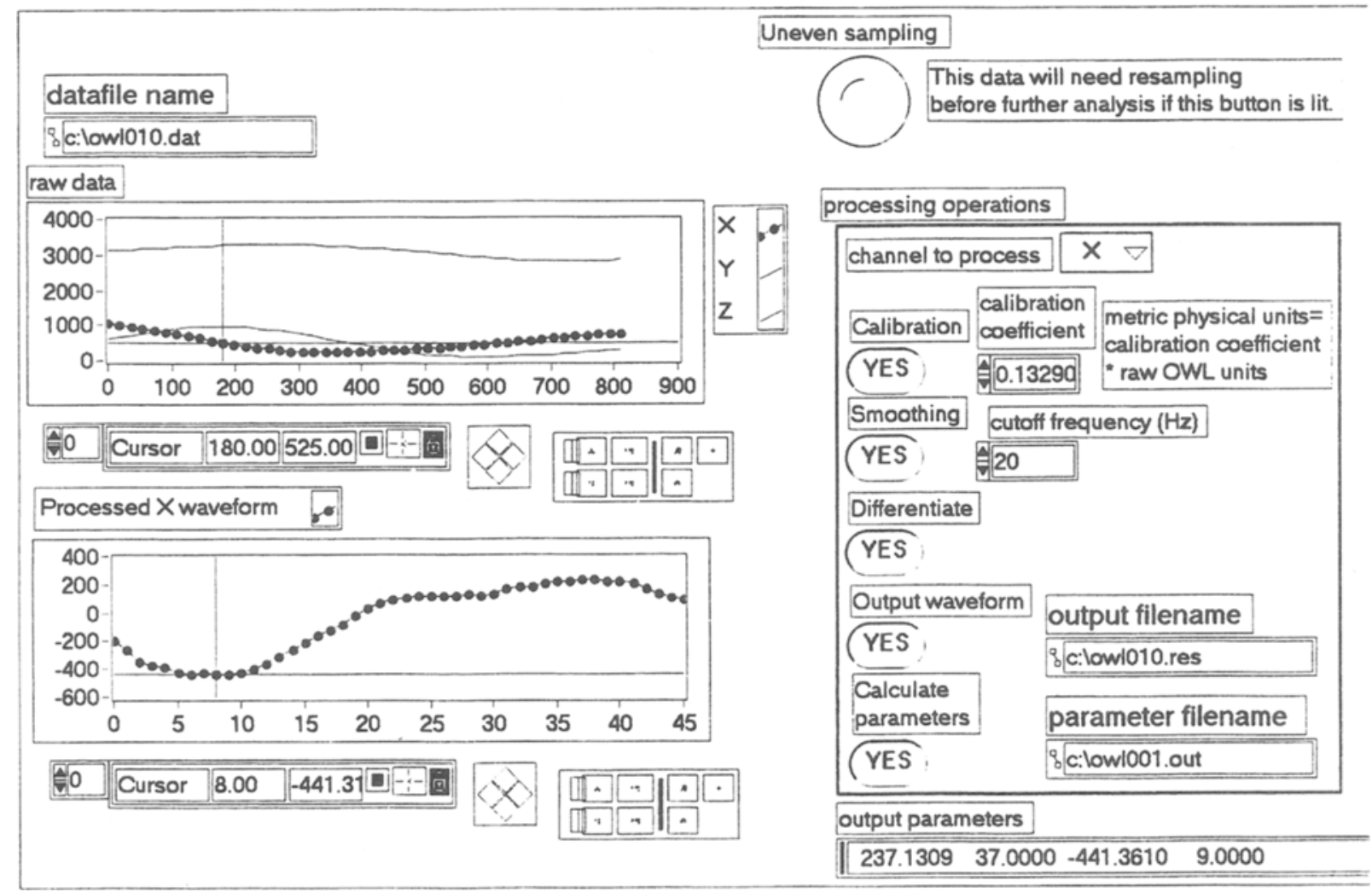

Figure 4. Front panel of a LabVIEW program for analyzing kinematic data. Note the succession of processing operations selected in the panel on right. 


\section{OWLSTAT.VI \\ 11/17/96 09:05 PM}

\section{Block Diagram}

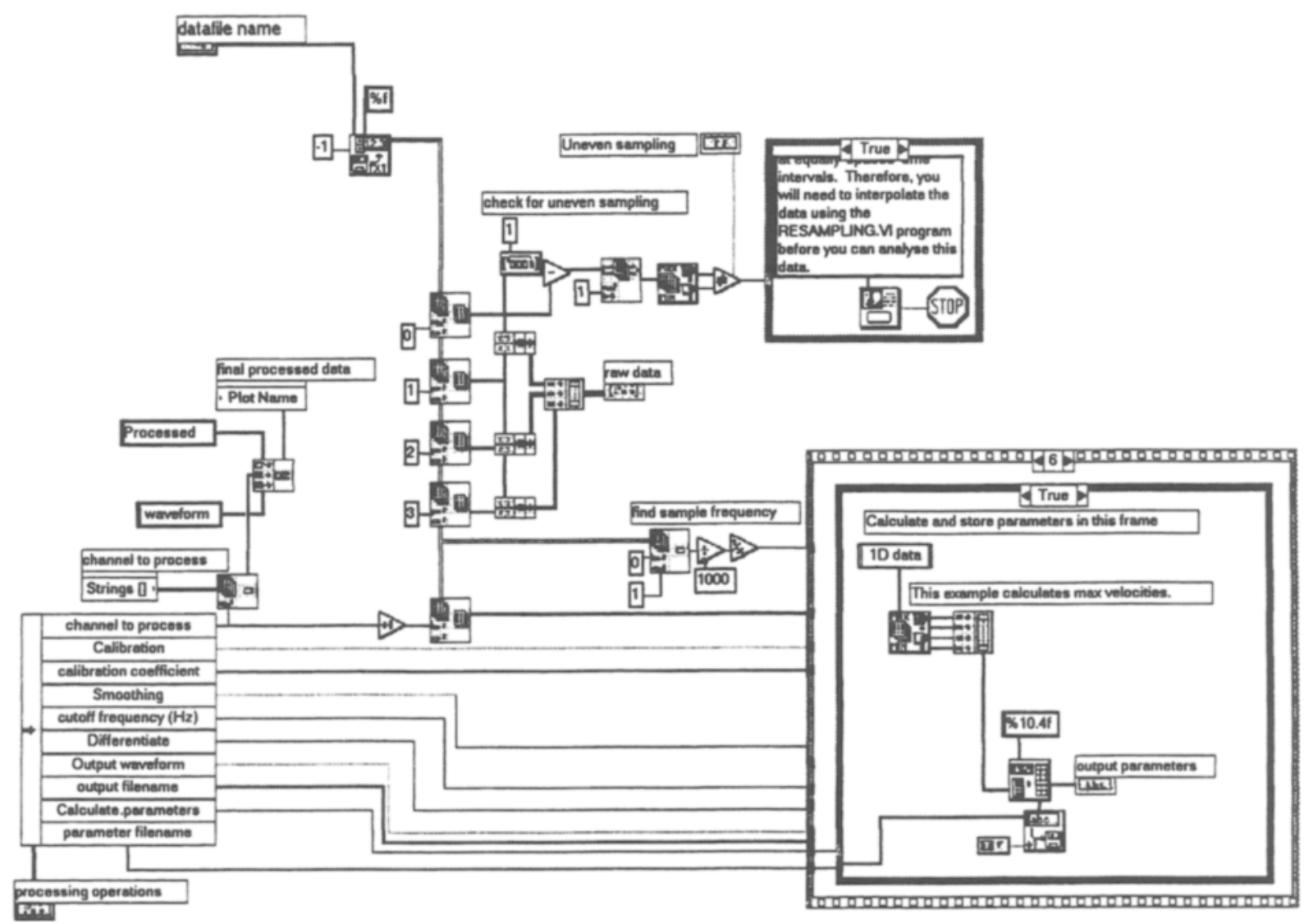

Figure 5. Adding an additional processing stage to the analysis program. This simply requires wiring the desired LabVIEW function so that it receives data from a global variable and it outputs results either back to the same global variable or, as in this case, to a file. This example calculates the maximum and minimum velocity of a movement and stores the result.

ming experience can add additional processing stages, calculate specific outcome measures from the kinematic traces, and store the results.

The front panel of this program is shown in Figure 4. The user enters a filename from which data are to be read and selects a range of processing options from the panel at right. These operations are then implemented in the order shown on the panel. The raw data read from the file are shown in the upper graph, and the final processed waveform is shown in the lower graph. The user has the option to store the processed data on disk for further analysis.

The signal processing operations performed are as follows. First, the time of acquisition of each sample (given in the first column of the data file) is checked to verify that all samples are equally spaced. Standard signal processing techniques cannot be applied if this is not the case; a warning indicator is therefore displayed, and execution of the VI is halted (unequally spaced data can be resampled using a resampling program, resample.vi). Perhaps the two most common operations in kinematic data analysis are smoothing and derivation of position signals. These are provided as options within the program. The smoothing is achieved by a dual-pass secondorder Butterworth low-pass filter, with a specified cutoff frequency. Derivation is achieved by a simple one-point difference algorithm, padded to provide appropriate initial and final conditions. Finally, the smoothed and/or derived waveform can be written to disk as a human readable spreadsheet file with a single column. As before, these files can be further analyzed within LabVIEW, Excel, or any other standard data manipulation tool.

\section{Design and Customization of Analysis Software}

Each movement experiment needs to be analyzed differently, and learning how to analyze kinematic data to answer the scientific questions at hand must be a major goal of any motor skills practical class. Therefore, the 
analysis software has been written in a way that allows easy customization and, in particular, easy addition of processing options to calculate outcome measures, such as peak velocities, movement times, and so on, from kinematic data. The design philosophy behind the analysis program is as follows. Each processing stage is requested or omitted by a yes/no button in the panel shown on the right. Processing stages operate in strict sequence, which should correspond to descending the options selected in the panel. A LabVIEW global variable is used to pass data through the processing stages, so that each frame of the sequence structure begins by reading from the global, performs transformations on the data, and then writes the processed data back into the same global. Adding additional analyses within this framework is therefore easily within the reach of the student programmer. As an example, the programmer is required to add an additional stage of analysis as shown in Figure 5. In the example, the value and location of the maxima and minima of the velocity trace shown in Figure 3 have been calculated. The only programming required is to connect the appropriate LabVIEW functions to the global data array and to build the resulting output measures into an array. This information is then written to a file in spreadsheet format for subsequent analysis.

\section{Applications}

We have found this system to be practical in most experiments in which movement of one point on the limb or body is to be tracked over a fairly short distance. These include measuring hand position in aimed movement, measuring head movements in orienting experiments (e.g., auditory localization), measuring body sway during posture, and measuring foot placement and movement during gait initiation. Simple experiments involving a small number of movements in a variety of conditions can be run using the software described above without further modification. For example, a Fitts's Law-type experiment can be run simply by running the runOWL program a number of times with a different target size and target distance on each occasion. Because the system is based on the fully functional programming language of LabVIEW. more complex experiments can easily be programmed. For example, visual stimuli can be displayed on the screen to guide or direct the current movement, and the PC's parallel port can be used to control additional external instruments via digital inputs and outputs.

\section{Supplier Details}

The OWL is made by Pegasus Technologies Ltd., Beit Panarama, 84 Ben Zvi Rd., Tel Aviv, Israel 68104. Tel: 9723518 2422. Fax: 9723518 2423. http://www. netvision.net.il/ peg

LabVIEW is made by National Instruments, 6504 Bridge Point Parkway, Austin, TX 78730-5039. Te1: (512) 794-0100. Fax: (512) 794-8411. http://www. natinst.com

Selspot is made by SELCOM AB, P.O. Box 250, S43325 Partille, Sweden. Tel: 46 (0) 313362510 . Fax: 46 (0) 31 446179. http://www.selcom.se

OPTOTRAK is made by Northern Digital Inc., $403 \mathrm{Al}-$ bert St., Waterloo, ON, Canada N2L 3V2. Tel: (519) 8845142. Fax: (519) 884-5184. http://www.ndigital.com

Fastrak is made by Polhemus Inc., 1 Hercules Dr., P.O. Box 560, Colchester, VT 05446. Tel: (802) 655-3159. Fax: (802) 655-1439. http://www.polhemus.com

Flock of Birds is made by Ascension Technology Corp., P.O. Box 527, Burlington, VT 05402. Tel: (802) 860-6440. Fax: (802) 860-6439. http://www.ascensiontech.com

\section{REFERENCE}

HAGGarD, P., \& WING, A. M. (1990). Assessing and quantifying the accuracy of measurements made with optical tracking systems. Journal of Motor Behavior, 22, 315-321.

\section{NOTE}

1. The LabVIEW virtual instruments can be downloaded from the directory http://www.psychol.ucl.ac.uk/patrick.haggard/OWL

(Manuscript received December 2, 1996; revision accepted for publication March 18, 1997.) 